FORMATION Formation emploi

Revue française de sciences sociales

150 | avril-juin 2020

Former aux "petits" métiers : regards internationaux

\title{
L'apprentissage pour « horizon » ou comment les élèves des classes populaires intériorisent le sens de leur orientation contrariée
}

Apprenticeship "on the horizon" : how pupils with working class backgrounds do internalize an upset sense of their school orientation

Eine Lehre für Horizont : wie die Schüler der Arbeiterklasse einen Sinn ihrer

Schulische verärgerte Orientierung verinnerlichen

El aprendizaje como "horizonte" o cómo los alumnos de las clases populares internalizan el sentido de su orientación frustrada

\section{Christophe Delay}

\section{OpenEdition}

Journals

Édition électronique

URL : https://journals.openedition.org/formationemploi/8118

DOI : 10.4000/formationemploi.8118

ISSN : 2107-0946

Éditeur

La Documentation française

Édition imprimée

Date de publication : 8 juillet 2020

Pagination : 27-54

ISSN : 0759-6340

Référence électronique

Christophe Delay, «L'apprentissage pour « horizon » ou comment les élèves des classes populaires intériorisent le sens de leur orientation contrariée », Formation emploi [En ligne], 150 | avril-juin 2020, mis en ligne le 02 janvier 2022, consulté le 06 janvier 2022. URL : http://journals.openedition.org/ formationemploi/8118; DOI : https://doi.org/10.4000/formationemploi.8118 


\title{
L'apprentissage pour " horizon » ou comment les élèves des classes populaires intériorisent le sens de leur orientation contrariée
}

\author{
Christophe Delay \\ Professeur en sociologie à la Haute école de travail social et de la santé Lausanne et Haute \\ école spécialisée de Suisse occidentale (HETSL/HES-SO)
}

Résumé

L'apprentissage pour « horizon » ou comment les élèves des classes populaires intériorisent le sens de leur orientation contrariée

Cet article étudie les choix d'orientation des élèves des classes populaires aspirant à entrer en apprentissage salarié en fin de scolarité obligatoire. Mobilisant une approche ethnographique, il montre comment s'intériorise dans la durée, au contact des enseignants en classe, puis lors d'un processus de socialisation professionnelle partielle et incomplète en stage, un sens de sa place. La confrontation des choix désirés à ceux finalement réalisés permet de saisir un important groupe d'aspirants contrariés dont la frustration variable est paradoxalement générée par le système scolaire helvétique, organisé en filières fortement hiérarchisées.

Mots clés : orientation scolaire-professionnelle, apprentissage, enseignement technique-professionnel, enseignement secondaire, enseignant, stage de formation, socialisation professionnelle, représentation de la formation, Suisse

Abstract

Apprenticeship "on the horizon": how pupils with working class backgrounds do internalize an upset sense of their school orientation

The aim of this article is to study the educational choices of youngsters who wish to enter apprenticeships at the end of compulsory schooling. Using an ethnographical approach, it shows how a sense of one's place is internalized by the pupils over the time, first: during everyday life interactions with teachers in classrooms and second: at work placement during a sketchy and incomplete process of professional socialisation. Confronting youngsters' ideal choices with their final real choices allows us to grasp an important group of upset aspiring pupils whose varying frustration is paradoxically generated by the Swiss education system which is divided in strongly differenciated school tracks. 
Keywords: school guidance \& vocational guidance, apprenticeship, technical $\&$ vocational education, secondary education, teacher, traineeship, professional socialisation, perception of training, Switzerland

Journal of Economic Literature: I 21

Traduction : auteur.

L'orientation des élèves des classes populaires vers l'enseignement professionnel a fait l'objet d'un regain d'intérêt, en France, cette dernière décennie. Plusieurs chercheurs ont montré les divers déterminants pesant sur ces choix.

Une première série de travaux insiste sur les parcours scolaires et sur les diverses attitudes et représentations des jeunes, productrices, in fine, de ces orientations. Willis (1977) a mis en évidence que l'entrée précoce dans le monde du travail résultait, chez les garçons, d'une "culture anti-école " qui conduisait à l'échec scolaire et était liée à la culture ouvrière d'atelier des pères ; néanmoins, l'importance, chez les apprentis, d'un rapport a priori négatif à la théorie qui donne sens à leur orientation, a depuis été confirmée (Moreau, 2003). La fonction de tri du système scolaire, qui divise par le redoublement, rend également beaucoup plus probable l'orientation vers l'enseignement professionnel pour ces élèves (Palheta 2012). Celle-ci découle encore de l'«observation ", par les jeunes, "de leur entourage pertinent" (Chauvel, 2011) ${ }^{1}$.

D'autres travaux se focalisent sur les ambitions parentales. Ainsi, le choix de l'apprentissage salarié est lié au "scepticisme" des parents quant à l'utilité de l'acquisition des diplômes généraux (Lemaire, 1996). Les coûts financiers élevés d'une poursuite d'études et les faibles chances de réussite seraient au fondement d'aspirations scolaires souvent moins élevées en milieu ouvrier (Boudon, 1984). Un différentiel d'ambition confirmé depuis ; en effet, alors que les cadres supérieurs visent le baccalauréat général pour leurs enfants, les ouvriers souhaitent plus souvent les engager dans la voie professionnelle (Brinbaum \& Kieffer, 2005).

L'influence des professeurs sur les orientations a fait l'objet de travaux moins nombreux. Si celle-ci a surtout été constatée lors des décisions de fin d'année (Palheta, op. cit.), quelques rares travaux ethnographiques ont mis en évidence le travail enseignant en amont des orientations.

1. En effet, ces derniers définissent un champ des possibles réaliste, à partir des expériences scolaires difficiles en formation générale qu'ils perçoivent ou qui sont rapportées par les aînés ou les amis dans leur entourage proche. 
Ainsi, les élèves des classes populaires de $1^{\text {re }}$ année des sections de technicien supérieur (STS) citent plus souvent leurs professeurs comme des personnes les ayant conseillés dans leur choix, les filières STS constituant, en dernière année de baccalauréat, un espace suggéré (Orange, 2013). Leurs aspirations faiblement constituées pèsent ainsi peu face aux conseils structurés des enseignants. Également indirectes, les injonctions professorales laissent un trouble sur d'autres filières (universitaires) non proposées. Elles produisent un sentiment d'évidence chez les élèves qui ne les envisagent pas comme possible scolaire. Ainsi donc, les élèves apprennent progressivement, par le biais de la parole professorale, à se faire à leur place à l'école. De telles influences apparaissent déjà au collège : les enseignants cherchent à convaincre les élèves les plus faibles que leur place est au sein de la voie professionnelle (Cayouette, 2016). Les séances d'éducation à l'orientation amènent ces derniers à apprendre à se situer dans cet univers et leur auto-exclusion des études longues s'opère avec la participation des enseignants.

À l'instar de ces travaux, notre enquête questionne la manière dont se façonne un "sens de l'orientation " dans le temps, chez les jeunes des classes populaires orientés, en Suisse romande, vers les filières préprofessionnelles de l'école secondaire inférieure. À la suite des travaux de Bourdieu, nous considérons que l'ordre social s'inscrit progressivement dans les esprits par le bais des rappels à l'ordre incessants qu'imposent les institutions (famille, système scolaire). "Les limites objectives deviennent sens des limites, anticipation pratique des limites objectives qui porte à s'exclure (...) de ce dont on est exclu» (Bourdieu, 1979, p. 549).

Ainsi, les orientations, loin de se construire uniquement lors des points de transitions en fin d'année et de provenir des "décisions individuelles » et "calculs " des élèves et de leurs familles (Boudon, 1984) résultent de "processus " construits par une "multiplicité d'acteurs" (Blanchard \& Cayouette, 2011).

Cet article montrera ainsi $1^{\circ}$ ) que les enseignants, dont la propension à s'arroger la responsabilité de l'orientation est plus forte en Suisse qu'en France - où celle-ci est plus subtilement partagée entre familles et système scolaire (Boudon, 1990) - constituent des agents de socialisation. En effet, en attribuant des "identités pour autrui " (Dubar, 2010) ils participent, chez certains élèves, à construire des orientations désirées vers l'apprentissage salarié ; 2) que d'autres acteurs plus périphériques - souvent peu considérés dans les analyses des travaux français privilégiant le travail de l'école et la famille (Palheta 2011 ; Blanchard \& Cayouette, op. cit.) - interviennent également dans ce processus. Ainsi, les maîtres de stages et patrons, plus impliqués dans les orientations en Suisse qu'en France - où l'apprentissage, moins développé, s'effectue davantage sous statut scolarisé - proposent une première forme partielle et incomplète de "socialisation préprofessionnelle " (Beaud \& Pialoux, 2003) et produisent également 
des jugements qui confortent les élèves dans l'intériorisation d'une identité pour soi de futur apprenti.

Finalement, afin de penser les choix comme des processus dont la construction s'effectue dans la durée (Blanchard \& Cayouette-Remblière, 2011) et les appréhender en actes (plutôt que rétrospectivement), les orientations désirées seront confrontées à celles réalisées. Il s'agit de saisir, à l'instar des travaux d'Orange (op. cit., p. 7), "les difficultés rencontrées dans leur réalisation". Cette mise en perspective permettra de mettre en évidence un groupe important d'aspirants contrariés.

L'article comprend quatre parties. La première montre le travail enseignant d'éducation à l'orientation en classe, qui attribue aux élèves une identité pour autrui de " futurs travailleurs salariés ». La seconde cherche à saisir les conditions d'adhésion des élèves aux discours enseignants. La troisième explore, à travers la réalisation des stages préprofessionnels, le renforcement, chez certains, du processus d'intériorisation d'une identité pour soi de "futur apprenti "; la dernière partie révèle que les orientations réalisées coïncident rarement avec celles désirées et qu'elles produisent une "identité d'apprenti contrariée ".

\section{Encadré 1. L'inégalité des chances scolaires en Suisse et à Genève}

En Suisse, la formation secondaire postobligatoire est au cœur du système des diplômes. C'est à ce niveau que l'on obtient son premier diplôme, unique pour certains. En fin de scolarité obligatoire (11e année), à 15 ans, les élèves choisissent entre des formations du secondaire supérieur générales ou professionnelles (CITE 3)(*). L'apprentissage salarié, dit dual, est la voie la plus choisie (60\% en 2011 : Wolter, 2014). L'accès à cette voie passe par des candidatures au sein desquelles les notes, ainsi que les stages réalisés au préalable, constituent des critères de sélection pour les patrons en vue de l'embauche. Les candidats retenus signent un contrat. Ils se forment au travail pratique en entreprise (durant trois à quatre jours par semaine) et à la théorie en école professionnelle (un à deux jours), en échange d'un salaire de quelques centaines d'euros. Cette formation permet l'obtention d'un Certificat fédéral de capacité (CFC), trois à quatre ans plus tard, et pour des élèves en difficultés scolaires, une Attestation de formation professionnelle (AFP) après deux ans (voir graphique 1). 


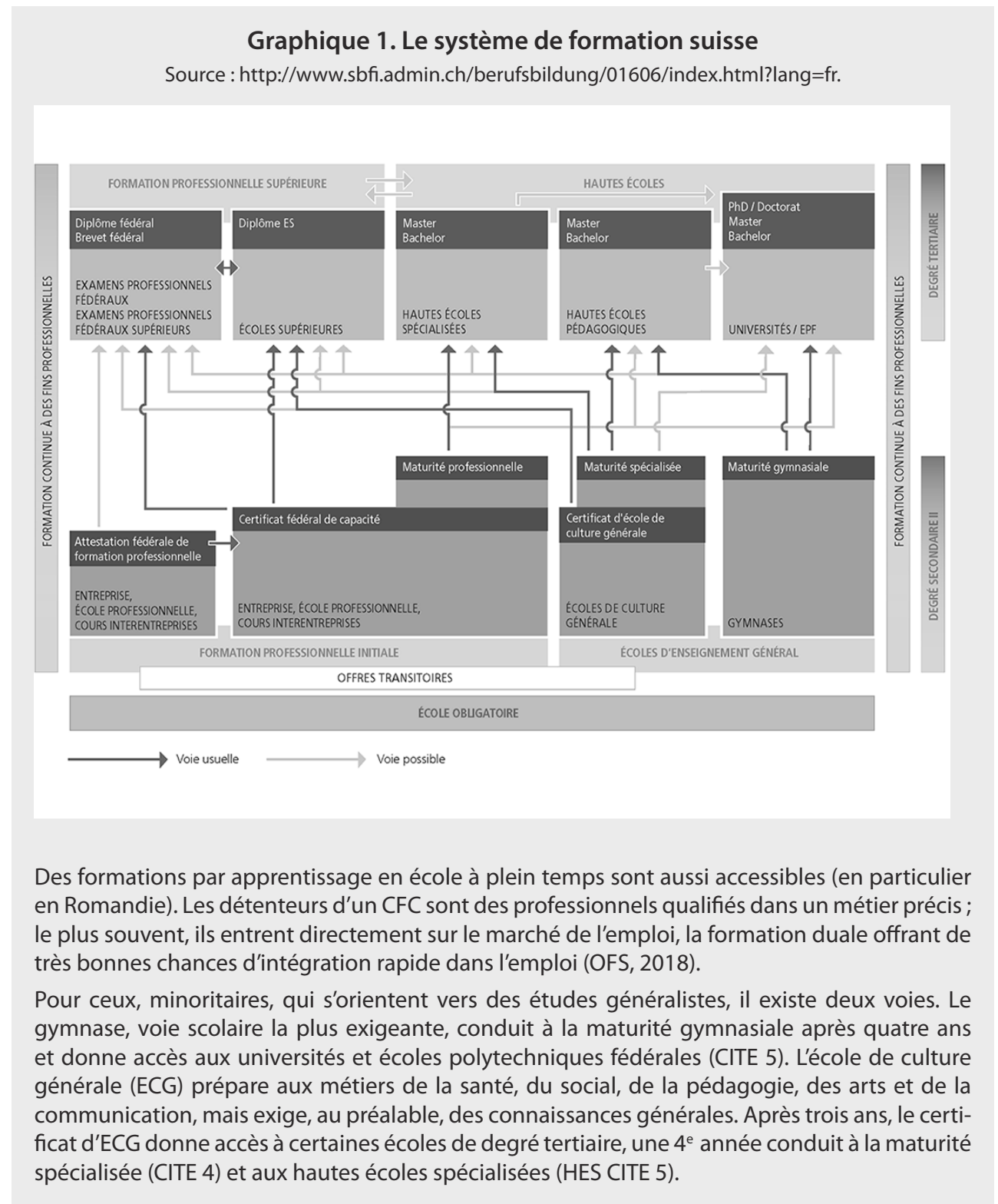


L'accès à ces diverses formations est régulé au niveau de l'enseignement secondaire inférieur (CITE 2). En effet, le système scolaire helvétique est très ségrégatif : à 12 ans, à la fin de l'école primaire, dans la majorité des cantons, il sélectionne les élèves dans deux à quatre voies inégalement prestigieuses, principalement selon leurs notes, mais aussi, et indépendamment des performances scolaires, en fonction de l'origine sociale, du sexe et de l'origine migratoire. Cela suggère une inégalité de traitement par l'école de dossiers scolaires équivalents (Felouzis \& al., 2015). Ces orientations sont largement irréversibles (Meyer, 2009). Plus souvent d'origine favorisée, les meilleurs élèves sont regroupés en filières pré-gymnasiales $\mathrm{A}$ - à « exigences étendues » - qui préparent aux études gymnasiales, puis universitaires, tandis que les plus faibles scolairement, plus souvent d'origine populaire et immigrée, sont orientés en filières préprofessionnelles B - « à exigences élémentaires » - donnant principalement accès aux formations professionnelles de niveau secondaire et, plus rarement, tertiaire.

Genève comporte un certain nombre de particularités. C'est le canton dans lequel les élèves se tournent majoritairement vers les études généralistes après l'école obligatoire $(58 \%$ : Wolter, 2018). Seul un quart se trouve en apprentissage salarié, la formation professionnelle étant perçue comme un second choix là où, en Suisse alémanique, elle occupe, dans l'opinion publique, une place égale dans la hiérarchie du système éducatif (Meyer, op. cit.). Cette spécificité tient notamment au fait que Genève, proche de la France, est influencée par le modèle de formation en école généraliste. Au moment de l'enquête, en 2012, l'accès au niveau secondaire supérieur dépend, dans la majorité des établissements, de la filière (A ou B) fréquentée ${ }^{(1)}$ à l'école secondaire inférieure, mais aussi, entre autres, de la promotion ${ }^{(2)}$ à l'issue de la $11^{\mathrm{e}}$, ainsi que de la moyenne générale annuelle. L'espace des formations disponibles y est hiérarchisé :

- Le gymnase est la voie la plus valorisée. Parmi les élèves de la filière $B$, seuls ceux qui ont obtenu les meilleures notes $y$ accèdent ${ }^{(3)}$. Toutes les autres options de formation leur sont ouvertes.

- L'école de commerce, formation professionnelle en école à plein temps la plus souvent choisie $\left({ }^{(4)}\right.$, prépare à un CFC en trois ans et conduit à des professions diverses et non à un métier spécifique comme d'autres CFC. Cette voie, ainsi que celle de l'école de culture générale (ECG) sont scolairement moins exigeantes, mais peuvent se poursuivre par une année de maturité (professionnelle ${ }^{(5)}$ ou spécialisée). Les élèves des filières $B$ admis doivent être promus et avoir de bons résultats scolaires. L'école de commerce constitue une option plus valorisante car les réorientations ultérieures vont de cette dernière vers l'ECG et autres apprentissages, et non l'inverse (Felouzis \& al., 2010).

- L'apprentissage salarié ne fonctionne pas uniquement selon des critères scolaires. En théorie, tout élève promu ou non qui a terminé sa scolarité obligatoire est admissible. Dans la réalité, nombre de professions organisent un test d'aptitude ${ }^{(6)}$. Bien que certaines branches requièrent un haut niveau d'exigences scolaires, l'apprentissage reste néanmoins moins valorisé car il offre de moindres perspectives de carrières (conduisant moins souvent en formation supérieure : Hupka \& al., 2011) et de progression salariale au long du parcours de vie (Korber \& Oesch, 2016).

- Enfin, les structures d'insertion sont accessibles aux élèves ne pouvant entrer directement en formation et devant parfaire leurs compétences scolaires durant un an. Les classes préparatoires, accessibles aux élèves promus, permettent, en cas de promotion, d'entrer en $1^{\text {re }}$ année d'école de commerce (EC) ou de l'ECG. Le Centre de transition professionnelle (CTP), accessible aux élèves non promus, forme ces derniers à la pratique en vue de l'apprentissage salarié. 
La réforme scolaire genevoise de 2013 a renforcé la sélectivité de l'école secondaire inférieure (l'accès à l'équivalent de la filière $A$ nécessite de meilleurs résultats), avec pour effet que davantage d'élèves sont regroupés dans les anciennes filières B. Le Conseil d'État cherche parallèlement à diminuer le nombre d'élèves optant pour les formations générales et, en remobilisant le secteur privé, mais aussi public, à « valoriser l'apprentissage » dans le canton de Genève. Cette promotion doit se faire à travers, notamment, une " amélioration du processus d'orientation et d'information ». II s'agit, pour les enseignants dispensant des cours d'information scolaire et professionnelle, de " mieux sensibiliser les élèves (notamment des ex-filières B) aux branches professionnelles » afin de "faciliter le passage des jeunes entre l'enseignement obligatoire et la formation duale» (DIP, 2015, p. 14).

${ }^{*}$ ) : Classification internationale type de l'éducation.

(1) La réforme scolaire de l'école secondaire inférieure a introduit, dès 2013, dans tous les établissements, trois regroupements en $9^{e}$ qui débouchent, l'année suivante, sur trois sections (LS : littéraire-scientifique ex-filière $A$; LC : langues vivantes et communication, ex-filière $B ; C T$ : communication et technologie, ex-filière B).

(2) Pour être promus, les élèves doivent avoir une moyenne générale d'au minimum quatre sur six.

(3) Pour les élèves en filière $A$, la promotion suffit. Les non-promus peuvent être admis à l'école de commerce ou à l'ECG.

(4) D'autres apprentissages scolarisés (technique) sont moins fréquentés.

(5) Si les titulaires d'un CFC (en apprentissage dual) peuvent également poursuivre par une maturité professionnelle, ceci reste beaucoup moins fréquent que pour les titulaires du diplôme de l'école de commerce (DIP, 2011).

(6) Portant sur les connaissances scolaires, les aptitudes et les motivations de l'élève.

\section{- Une enquête ethnographique dans un établissement scolaire genevois}

Cet article s'appuie sur les résultats d'une enquête ${ }^{2}$ menée dans un établissement scolaire secondaire inférieur d'un quartier populaire de Genève.

Nous avons cherché à saisir, parmi les élèves de cet établissement, en $11^{\mathrm{e}}$ et orientés en filière B (filières préprofessionnelles), les déterminants, mais aussi le sens assigné aux orientations dans les diverses formations qui leur sont accessibles. Trois classes nous ont été ouvertes par des enseignants connus d'un collègue de travail. Durant six mois, dès le $2^{\mathrm{e}}$ trimestre, nous avons observé les attitudes des 40 écoliers et leurs interactions avec l'enseignant en cours d'information scolaire et professionnelle (ISP) visant à les préparer à se pré-inscrire dans les diverses filières de l'école postobligatoire. De nombreuses discussions formelles $(\mathrm{N}=7)$ avec les enseignants, pour comprendre la logique présidant aux orientations conseillées, mais aussi informelles, après les observations, ont permis de recueillir des propos en situation.

2. Financée par le Fonds national de la recherche scientifique Suisse (2012-2013). 
Un rapport de familiarité ayant été établi par une présence bi-hebdomadaire en classe, nous avons demandé, lors d'un cours, la participation de ceux qui le souhaitaient à un entretien, trois mois avant les choix définitifs, au moment où les destins scolaires sont quasiment scellés. Vingt-cinq élèves aux scolarités variées, mais majoritairement "en grandes difficultés " ${ }^{3}$ (Rastoldo \& al., 2007) - et appartenant essentiellement aux milieux populaires - se sont portés volontaires. Les entretiens, d'une durée d'une heure à une heure et demie abordaient les thématiques des parcours scolaires juvéniles antérieurs, du rapport à l'école et aux enseignants, des choix de formation au niveau secondaire supérieur et des projections dans l'avenir. Ils ont eu lieu dans une salle de classe où nous avons cherché à créer une atmosphère conviviale ${ }^{4}$. Ainsi, certains acceptaient l'échange, y voyant l'opportunité de manquer une heure de cours ou de réfléchir à leur orientation ou encore d'utiliser notre réseau pour les aider à trouver un stage.

L'échantillon n'est pas représentatif de la population étudiée : les élèves n'ayant aucune idée d'orientation en tête ont probablement été davantage disposés à décliner la demande, s'estimant incapables de présenter un "moi acceptable " (Mauger, 1991) 5 . Par ailleurs, par rapport à l'ensemble des élèves des trois classes investiguées, notre échantillon comporte davantage d'élèves en grande difficulté ${ }^{6}$ et moins d'élèves en difficulté moyenne : il est possible que les élèves aux scolarités meilleures aient été peu enclins à perdre deux périodes de cours pour s'entretenir avec nous. Notre échantillon reste cependant pertinent au vu de la problématique étudiée : la surreprésentation des élèves en grande difficulté facilite en effet l'analyse des déterminants de l'orientation des classes populaires.

Les entrevues coïncidaient avec l'injonction scolaire faite aux élèves à se pré-inscrire dans l'une des voies de formation du secondaire supérieur (en indiquant un choix " possible » et un choix "idéal »). Les matériaux utilisés ici sont tirés des entretiens réalisés avec les neuf élèves - six garçons et trois filles, presque tous d'origine étrangère - ayant opté pour le choix idéal de l'apprentissage salarié, l'option la plus souvent désirée au sein de notre échantillon au vu de la surreprésentation des élèves en " difficulté " ${ }^{7}$. Il s'agit ici de saisir à quelles conditions les élèves adhèrent aux discours enseignants et quelle est l'influence spécifique de ces discours sur leurs désirs. La participation aux cours d'ISP ( $\mathrm{N}=13$ en majorité en filière $\mathrm{B})$, aux conseils de classe à fin mars - puis en fin de parcours en juin $(\mathrm{N}=6)$ permet de saisir - en plus des entretiens

3. Soit 19 élèves pour six se trouvant "sans difficultés " $(\mathrm{N}=3)$ ou en "difficultés moyennes " $(\mathrm{N}=3)$.

4. En proposant aux élèves à boire et à manger.

5. En effet, les enquêtés n'acceptent de se prêter à la situation d'enquête que s'ils pensent que l'enquête les " grandira " plutôt qu'elle ne les rappellera à leur « petitesse ». Le refus d'engager la conversation s'impose alors dans ce dernier cas.

6. Soit $76 \%$ des élèves interviewés (la moitié promus $\mathrm{N}=9$, l'autre non promus $\mathrm{N}=10$ ) alors qu'ils représentent $68 \%$ de tous les 40 élèves observés dans les trois classes.

7. En effet, seuls six visent l'ECG, six une école professionnelle à plein temps et quatre le gymnase. 
réalisés à un moment ponctuel - les aspirations juvéniles dans la durée, afin de voir dans quelle mesure elles évoluent et parviennent ou non à se réaliser.

L'analyse des matériaux visait à connecter les expériences des interviewés avec des catégories plus larges (appartenance de classe, de sexe). En concordance avec l'approche bourdieusienne, nous avons considéré que les choix des élèves résultaient de dispositions de l'habitus (envers l'école, l'avenir) et dérivaient de multiples instances de socialisation (famille, professeurs, pairs) et de conditions d'existence spécifiques. Les matériaux présentés ci-après résultent d'analyses jointes, issues de différentes sources de données qui ont été triangulées de manière à produire des inférences : 1) des extraits sélectionnés d'observations annotées dans lesquelles le travail enseignant d'éducation à l'orientation est décrit et les aspirations juvéniles discutées/confrontées en classe ; 2) des extraits d'entretiens conduits avec les aspirants apprentis.

\section{Le travail enseignant d'éducation à l'orientation : attribuer une identité de « travailleur salarié » aux élèves}

L'observation des cours d'information scolaire et professionnelle in situ permet de saisir le travail enseignant réel d'éducation à l'orientation.

Les deux sous-parties suivantes permettent de montrer comment, tant par la parole que par les silences, les enseignants préparent les élèves à choisir des formations différenciées (longues ou courtes) selon la filière fréquentée (1.1), mais aussi comment ils proposent, en filière préprofessionnelle $(\mathrm{B})$, des réorientations aux élèves scolairement ambitieux et "sans difficultés ", au principe des révisions à la baisse des ambitions initiales des élèves des milieux populaires (1.2).

\subsection{Faire advenir le probable}

Pour les élèves en filière A (filière pré-gymnasiale), les enseignants précisent, lors des cours qui ont lieu toutes les deux semaines, que la construction des CV "n'est pas ce qu'il y a de plus important pour [eux] à présent". Ils sont mis en situation par le biais de petits emplois renvoyés à un futur lointain, à la fin des études, ou auxquels ils pourraient postuler durant les vacances d'été. Les enseignants anticipent le type d'emplois auxquels leurs élèves pourraient postuler, en indiquant des professions valorisées socialement. Les modèles de lettre de motivation travaillés en classe sont fictifs, sans lien avec des postes actuellement vacants permettant de mettre les élèves en situation concrète. Les élèves ne sont pas non plus incités à chercher des stages, au prétexte qu'ils auront le temps, ultérieurement, de se poser de telles questions.

Toute autre est l'ambiance régnant en filière B (préprofessionnelle). Conformément au plan d'action du Conseil d'État, les enseignants viennent en cours avec des offres de 
places d'apprentissage, disponibles et téléchargées sur des sites officiels, pour mettre les élèves en situation concrète. Ils contribuent, lors des exercices en classe, à faire advenir le probable à moyen terme, entendu comme les " probabilités objectives scientifiquement construites (chances d'accès à tel ou tel bien ${ }^{8}$ )" (Bourdieu, 1980, p. 90), en projetant les élèves dans des métiers sexués peu valorisés socialement, leur attribuant une double identité (pour autrui) de sexe et de classe (Dubar, op. cit.) :

"Enseignant: Admettons, Amina, tu veux faire coiffeuse, qu'est-ce qui pourrait influencer ce choix?"

"Une élève (père manœuvre, mère au foyer, non promue) : la personnalité?"

"Enseignant: Tout à fait, je ne verrais pas Amina assise derrière un bureau face à un ordinateur toute la journée. " [Rires dans la classe]"

Les exercices en cours qui ont lieu chaque semaine visent à rappeler à tous les élèves (même aux quelques meilleurs d'entre eux) que les choix s'opèrent dans un champ des possibles scolaires restreint, et les enseignants les invitent implicitement à réfléchir à des métiers précis :

"Enseignant : Est-ce que toutes les portes sont ouvertes concernant vos choix ?»

"Une élève (mère au foyer, père décédé) : Ben non, on est en B."

"Enseignant: La réalité des notes, voilà un facteur qui influence vos choix de professions."

Certes, la moitié d'entre eux étant non promus au $2^{\mathrm{e}}$ trimestre, les enseignants cherchent donc à les ramener vers les orientations " possibles", comme le souligne un enseignant: "Il y a une réalité qui n'est pas acquise chez eux». Mais loin de s'en tenir à une confrontation des élèves « au réel ", ils visent à préparer les élèves au " probable », en tenant compte de la filière à laquelle ils sont assignés et de leur potentiel supposé/crédité.

Ainsi, quatre élèves interviewés (d'origine étrangère, de milieux populaires) sont promus en fin d'année et pourraient, de ce fait, viser à moyen terme le gymnase pour accéder au métier souhaité ou satisfaire les désirs parentaux. Pourtant, les enseignants omettent de parler des " passerelles » qui permettraient à ces élèves et aux promus n'ayant pas redoublé par le passé de rejoindre la $11^{\mathrm{e}}$ filière $\mathrm{A}$, par redoublement promotionnel, afin de raccrocher le gymnase. La majorité d'entre eux se conforme ainsi à la structure contraignante des filières qui, pour les élèves en $B$, est censée avant tout les préparer aux formations professionnelles ${ }^{9}$. Ils cherchent ainsi à éviter aux élèves promus ce qui est anticipé comme un

8. En fonction de l'appartenance de sexe, mais aussi, en particulier, de l'appartenance de classe des élèves : le diplôme du secondaire II des élèves de milieu ouvrier, le plus souvent achevé, est le CFC d'apprenti dual ( $45 \%$ de la population, contre $19 \%$ des élèves des classes supérieures ; voir DIP, 2009).

9. Une certaine proportion de l'ensemble des élèves des filières $11 \mathrm{~B}$ à Genève s'oriente vers les filières générales (ECG : $22 \%$; gymnase : $3 \%$ ). Cependant, ces orientations restent moins empruntées que les autres filières professionnelles ( $24 \%$ vont en école à plein temps et $9 \%$ en apprentissage salarié), sans compter que parmi les $42 \%$ de jeunes orientés vers les structures d'insertion, une part considérable se prépare de toute 
échec programmé, conduisant à de douloureuses révisions ultérieures à la baisse des ambitions, fondé sur l'expérience enseignante, sur des évaluations annotées souvent «indulgentes » et la connaissance des statistiques, comme l'explique une enseignante :

"Pour avoir constaté des doublages, après ils vont en $A$, pis c'est guère mieux, tu vois, ils ont de la peine, alors des fois, ils arrivent finalement à passer au gymnase, mais ils font partie des $70 \%$ d'échec en première année parce qu'ils y arrivent pas. "

Dès lors, les remarques professorales répétées en classe cherchent à rendre l'orientation vers le gymnase " a priori impensable ».

De même, ne sont pas évoquées les orientations possibles (maturité, degré tertiaire) après certaines formations secondaires en école à plein temps, formations auxquelles certains élèves se destinent par le biais de la passerelle " préparatoire ». En fait, que cela soit dans l'optique de la revalorisation de la filière duale ou pour préserver les élèves de l'échec anticipé, les enseignants anticipent qu'ils trouveront un apprentissage salarié en cours de route. C'est ce que confie une enseignante qui doute et s'inquiète de l'orientation vers la préparatoire de l'ECG d'une élève promue (père ouvrier, mère aide comptable, portugais) désirant devenir éducatrice de jeunes enfants :

"Effectivement, elle a les notes, mais a-t-elle le niveau pour y rester? Ici en B, elle l'a, mais quand elle sera mélangée avec d'autres élèves des filières $A$, l'année prochaine, je ne sais pas. C'est ça qui nous inquiète" ".

Les statistiques indiquent qu'une part substantielle des élèves en préparatoire ECG ou de l'école de commerce se retrouvent, trois ans plus tard, en apprentissage salarié ; cependant, entre 20 et $30 \%$ des élèves parviennent à se maintenir dans la durée dans ces filières scolaires (Hrizi \& al. 2014). Dès lors, les enseignants ne tendent-ils pas à sous-estimer les possibilités de certains élèves à poursuivre des études du fait de leur origine étrangère et défavorisée (voir aussi Bolzman \& Perregaux, 2008) ?

Les filières s'apparentent ainsi à des " tunnels » dans lesquels les jeunes s'engagent et qui les conduisent d'un point à un autre (vers une formation postobligatoire attendue) et où le passage vers une filière plus élevée (ou basse) conduisant à d'autres formations ne devrait être emprunté qu'exceptionnellement (voir aussi Gomensoro \& Bolzman, 2015).

\subsection{Réorienter}

En classe, les élèves en filière B sont incités à effectuer des stages. Les informations qui circulent à ce sujet ont trait à la recherche d'apprentissage, au recrutement en direct qui s'effectue à l'Office d'orientation professionnelle. Les enseignants qui fondent leurs attentes sur des "indicateurs de valeur scolaire " (résultats antérieurs, filières ": Duru-

évidence à la formation professionnelle en entrant soit au CTP, soit en préparatoire de l'école de commerce (données DIP pour 2012, non publiées). 
Bellat, 2002, p 124) suggèrent aux rares élèves « sans difficultés » des filières B - qui souhaitent entrer au gymnase, ont les notes ou le potentiel pour y accéder - des orientations vers l'apprentissage salarié (ou l'ECG). Ils cherchent dès lors à "faciliter le passage des jeunes entre l'enseignement obligatoire et la formation duale en évitant les transitions inutiles, onéreuses et parfois décourageantes ", conformément au plan d'action du Conseil d'État (DIP 2015, p 15).

Ils engagent alors un travail de "conversion des aspirations professionnelles » (Zunigo, 2008) des élèves des classes populaires. Ainsi, Michel (père employé de bureau), descendu de $10^{\mathrm{e}} \mathrm{A}$ en $11^{\mathrm{e}} \mathrm{B}$, meilleur élève de sa classe et consacrant beaucoup d'efforts pour obtenir des notes lui permettant d'entrer au gymnase, se voit proposer l'option suivante : "Il y a une séance d'information à l'office d'orientation sur la finance, ça peut te concerner?". Ces remarques ne sont sans doute pas pour rien dans la révision, quelques mois plus tard, de son choix d'entrer en école de commerce. Ainsi, l'observation de la révision à la baisse des aspirations entre le $2^{\mathrm{e}}$ et $3^{\mathrm{e}}$ trimestre de deux bons élèves, ainsi que les propos critiques des trois élèves sans difficultés aspirant à entrer au gymnase, à propos des orientations fortement déconseillées par les enseignants ${ }^{10}$, suggèrent que dans chaque filière, toute personne cherchant à s'écarter de la trajectoire attendue et programmée est réorientée vers ladite filière (voir aussi Gomensoro \& Bolzman, op. cit.).

\section{Les conditions sociales d'adhésion juvénile au discours enseignant}

La récurrence des commentaires enseignants lors des exercices effectués en classe produit des effets sur la majorité des élèves de notre échantillon, qu'ils souhaitent "s'en sortir " par l'école ou " en sortir", puisque rare sont ceux $(\mathrm{N}=6)$ qui se projettent dans les études longues (Delay, 2018).

Les élèves les plus enclins à chercher des stages et à cocher le choix idéal de l'apprentissage salarié ont des profils spécifiques par rapport à ceux qui refusent ou se montrent indifférents à cette assignation d'identité. Ils ont ainsi plus souvent redoublé une année, sont arrivés en Suisse après la naissance, ont des " difficultés moyennes " ou " grandes ", connaissent plus souvent, dans leur entourage, des apprenti.e.s, mais aussi ont plus souvent des parents ouvriers ou employés qualifiés (à faible capital scolaire), aux configurations familiales précaires (divorcés/remariés) et à fécondité large. Ceux qui résistent aux injonctions professorales (parmi eux, plusieurs sont en "grande difficulté ") ont plus souvent des scolarités linéaires, des proches en études longues et des parents plus

10. Comme l'explique Nassim, dont les notes lui permettent d'entrer au gymnase : "On m'encourage pas d'aller au gymnase. C'est pas qu'ils m'aiment pas, c'est juste savoir qu'on est en B, on a un retard (pour eux). On me dit surtout d'aller à l'ECG». 
stables qui possèdent des diplômes et ont des aspirations scolaires plus élevées (voir tableau 1 en annexe de la version électronique).

La mise en évidence de deux cas permet de saisir quelle est l'influence socialisatrice spécifique des enseignants dans le choix de l'apprentissage, ainsi que le "sens " que les jeunes associent à leur orientation. Au moment de l'entretien, ces derniers se trouvent dans une situation scolaire leur permettant d'opérer un choix, ce qui signifie que plusieurs options sont (au moins) théoriquement envisageables pour eux ${ }^{11}$.

\subsection{Un choix évident revendiqué}

Manuel a 15 ans (origine brésilienne, beau-père horloger indépendant, école professionnelle ; mère au foyer, scolarité obligatoire ; deux sœurs aînées) et éprouve, au moment de l'entretien, des "difficultés moyennes ". Il revendique le choix évident d'entrer en apprentissage salarié :

"Je suis tellement sûr de ce que je voulais, ils [mes parents] se sont dit "ouais de toute façon c'est évident"."

Au vu de ses notes, il pourrait cependant entrer en école de commerce ou à l'ECG. Sa scolarité a été entachée de difficultés qui s'expliquent moins par la trajectoire migratoire l'ayant conduit à intégrer en cours de route, à huit ans, le système scolaire suisse (il n'a pas rencontré de difficultés à apprendre le français) que par une "culture anti-école " à composante ouvrière, proche de celle décrite par Willis (op. cit.), et sans doute issue d'une socialisation familiale qui a conduit à un redoublement en fin d'école primaire :

"J'ai refait ma 8e. Ça s'est très mal passé. Je faisais rien. Je m’embrouillais avec d'autres gars, ça cognait, avec ma prof, ça passait mal, je lui piquais des craies, j'ai été écrire partout avec des potes "c'est une grosse salope", je m'en foutais, l'école, c'est de la merde."

Entré en $9^{\mathrm{e}} \mathrm{A}$, il continue à adopter une culture oppositionnelle et sera réorienté en $11 \mathrm{~B}$ sans en éprouver de malaise, marquant une indifférence scolaire durable. Invité par les enseignants à effectuer des stages, ces discours ont généré chez lui un « sens des limites " déjà façonné durant l'enfance par sa socialisation familiale antérieure. Manuel a en effet grandi au Brésil au contact de son grand-père, dans son atelier d'horlogerie, pendant que sa mère, divorcée, occupait des emplois peu qualifiés. Il se rappelle l'avoir côtoyé et avoir acquis, lors d'un processus de socialisation par pratique directe et expérimentation (Lahire, 2002), des « dispositions pour le travail manuel» :

"Il me montrait tellement volontiers [comment réparer les montres] que ça se voyait que c'était un truc qu'il adorait faire."

11. Sur les neuf élèves aspirants apprentis, six ont des notes permettant d'envisager l'alternative d'une entrée en école à plein temps (en préparatoire ou directement en $1^{\mathrm{re}}$ ). 
L'éducation professorale à l'orientation génère, en réactivant les souvenirs liés à cette première socialisation informelle dans un cadre professionnel, un sentiment d'évidence ; en effet, durant le $2^{\mathrm{e}}$ trimestre, Manuel choisit d'effectuer un stage dans un centre de formation professionnelle (pôle technique CFPT) et se trouve tout de suite " à sa place " dans ce lieu :

"Comme dans ma famille, il y a eu des antécédents horlogers, je me suis dit "je vais essayer", pour voir si ça me va. J'ai fait le stage au CFPT et j'ai tout de suite croché. Je me suis senti bien là-bas dans l'école, tout de suite chez moi."

Manuel envisage d'autant moins l'option des études longues qu'il trouve par ailleurs dans son entourage proche des exemples familiaux ayant effectué des scolarités courtes et auxquels il s'identifie fièrement :

"C'est un truc de famille, mes oncles ont arrêté l'école dès que l'école obligatoire était finie. Mon cousin (apprenti mécanicien automobile), c'est pire que moi (à l'école). On a toujours préféré grandir, et aller directement travailler pour gagner sa vie. "

Ce choix de l'évidence se trouve encore renforcé par la faible aspiration scolaire de son père, resté au Brésil, et par l'invitation faite par son beau-père de suivre un autre stage dans sa petite entreprise, en lui faisant miroiter l'idée d'une collaboration future avec lui ("il m’en a déjà parlé, "ouais dans le futur, si ça te plait vraiment, on peut faire associés" "). Sa mère, elle, est fière que son fils reprenne l'héritage familial ouvrier. Ce choix prend également sens comme moyen d'adhésion au "modèle du jeune consommateur" (Moreau, op. cit.), tout en s'ancrant dans les conditions d'existence des classes populaires (voir aussi Pasquali, 2010). Le jeune homme vit en effet dans un petit appartement où son beau-père a divisé le salon pour créer une chambre pour lui ; le salaire d'apprenti lui permettrait ainsi de ne pas peser sur un budget familial modeste :

"Rien qu'en me responsabilisant un peu plus vis-à-vis de l'argent, acheter mon abonnement, quelques habits. Ça va déjà un peu soulager les épaules de ma mère, tu vois."

Dans son cas, la socialisation, scolaire et familiale, joue un rôle de "renforcement " (Darmon, 2006) ${ }^{12}$ de ses aspirations et produit un choix revendiqué et vécu comme évident. Après l'obtention de son CFC, Manuel s'imagine avoir un emploi stable et une position confortable.

\subsection{Un second choix prudent}

Mirela a 15 ans (origine kosovare, père chauffeur de taxi, ancien maçon, scolarité obligatoire ; mère au foyer, école secondaire supérieure générale, deux frères aînés) et

12. Parler de socialisation de renforcement, c'est considérer qu'une instance de socialisation postérieure (ici l'école) vient prolonger l'influence d'une socialisation antérieure (ici familiale) pour exercer des effets fixateurs sur l'individu (au niveau de son système dispositionnel, ici les aspirations éducatives). 
est une élève avec des "difficultés moyennes » vu ses notes. Le choix de l'apprentissage salarié relève, chez elle, moins d'une évidence que d'un choix prudent issu d'une révision à la baisse de ses ambitions. Arrivée du Kosovo en Suisse à l'âge de deux ans, avec sa mère venue rejoindre une première fois le père de Mirela, qui travaillait sans permis dans le bâtiment, elle est contrainte, au vu de la précarité de la situation de son père, de repartir avec sa mère un an et demi plus tard. Avec sa mère, elle rejoindra son père une seconde fois à l'âge de dix ans, lorsque sa situation s'est stabilisée (ce dernier est désormais bénéficiaire d'un permis de travail $B$, renouvelable chaque année). Mirela a alors intégré rapidement une classe d'accueil avant d'entrer en $9^{\mathrm{e}} \mathrm{B}$. Sa facilité à ré-apprendre le français après un premier séjour lui vaut d'effectuer une scolarité linéaire et de commencer sa $11^{\mathrm{e}}$ avec de très bons résultats permettant de viser le gymnase. Pourtant, ce choix ne figure pas dans son champ des possibles scolaires - alors même qu'elle avait caressé l'idée d'un métier artistique de cinéaste dans un premier temps - car elle a intériorisé l'interdit professoral de cette voie réservée aux élèves des filières $\mathrm{A}$ :

"Je ne savais pas trop si je pouvais aller au gymnase parce que je sais que c'est très dur." Ayant intériorisé une "disposition prudente " ${ }^{13}$ populaire et féminine (Baudelot $\&$ Establet, 1992), elle privilégie le choix plus réaliste de l'école de commerce. Cette voie d'ascension est en effet souvent empruntée par les filles issues de la migration (Bolzman \& al,. 2003) car elle offre un diplôme professionnel tout en permettant de poursuivre des études ${ }^{14}$ :

"L'année passée, j'étais très forte en maths, je voulais vraiment aller à l'école de comm. En plus, j'avais un CFC à la fin."

La baisse de ses notes en maths, durant le $1^{\text {er }}$ trimestre $\left(11^{\mathrm{e}}\right)$, alors qu'elle travaille durement, produit une démotivation scolaire. D’une part, il y a les conseils de ses amis ayant rencontré des difficultés dans cette voie qui lui font anticiper une difficulté dans cette filière ; d'autre part, ceux, directs, de son enseignant, qui lui propose la filière générale féminisée dans le domaine du care, comme sas d'attente de l'apprentissage ${ }^{15}$, plutôt que la filière plus mixte de l'école de commerce ("il [le prof] m’a dit que je devrais mieux faire d'aller à l'ECG»). Tous ces conseils l'ont convaincue de revoir

13. Notre recherche s'inscrit dans une sociologie dispositionnelle qui postule que toute disposition - au principe de la diversité des pratiques des individus - est le produit incorporé d'une socialisation passée et se constitue dans la durée, dans la "répétition d'expériences relativement similaires» (Lahire 2002, p. 20).

14. 18 mois après avoir achevé l'école de commerce, $49 \%$ des élèves poursuivent une formation ; $33 \%$ sont en emploi (DIP, 2011).

15. L'année préparatoire à l'ECG constitue, selon la brochure "Après la scolarité obligatoire ", distribuée à tous les élèves, "une bonne préparation à l'apprentissage ". En effet, suite à cette année, $12 \%$ entrent en apprentissage salarié (Hrizi \& al., op. cit.). Si l'obtention du certificat ECG conduit $68 \%$ des élèves à se trouver, 18 mois après, en formation, parmi eux, $20 \%$ sont en apprentissage salarié (DIP, 2011). 
ses ambitions à la baisse et de viser un apprentissage salarié, alors même qu'elle est consciente d'avoir les notes pour entrer à l'école de commerce :

"Mes amis mont dit que c'était trop dur [école commerce], donc j'ai choisi plutôt l'ECG et là, vu que je peux pas avoir de CFC et que je dois continuer mes études, là je veux pas, sans CFC, on peut pas travailler, je veux avoir un CFC et travailler. Mais j'ai un niveau fort qui me permet d'aller en $1^{r e}$ de l'école de comm'."

Le conseil enseignant d'entrer à l'ECG - plutôt que de proposer un redoublement promotionnel auquel elle a droit étant donné sa trajectoire linéaire et qui permettrait de rejoindre la filière A pour viser le gymnase - paraît exprimer une "représentation stéréotypée genrée " (Duru-Bellat, 2008). En effet, les enseignants attendent par exemple "davantage d'une fille des qualités d'amour pour les enfants" (Guilley \& al., 2014). Il dénote également la tendance des enseignants à sous-estimer le niveau de compétence des enfants d'ouvriers en anticipant davantage d'échecs de leur part (voir aussi Duru-Bellat, 2002). En effet, à son camarade Alfredo, d'origine chilienne et de parents appartenant à la classe moyenne (père travailleur social ; mère assistante médicale), le même enseignant propose l'école de commerce, alors que ses résultats en chute libre sont bien moins bons.

Les hésitations de Mirela - à l'opposé du sentiment d'évidence de Manuel - se lisent également comme une difficulté, chez les filles, à trouver, au sein de leur famille, un modèle d'identification, sa mère étant au foyer et son père un ancien ouvrier en ascension sociale. Pressée, en cours d'éducation à l'orientation, de trouver des stages, Mirela procède par une recherche active qui l'éloigne du monde scolaire et accroît ses difficultés. L'acceptation de cet horizon du probable est renforcée par les discours parentaux qui l'incitent à se frotter au monde professionnel ("ils mont conseillé de faire un stage, voir comment ça se passe") en s'appuyant sur les expériences professionnelles (dans le médical) de plusieurs proches (femmes) de la famille.

Elle s'explique aussi par l'expérience de l'échec scolaire qu'elle rencontre comme "destin banal " (Bourdieu \& Passeron, 1985) autour d'elle, en voyant les parcours de ses frères ainés tous entrés au CTP. Comme Manuel, Mirela se projette à l'avenir dans le monde du travail. Sa prudence semble typique des filles qui «intériorisent jusqu'à l'excès les normes scolaires" et "ont du mal à simposer dans les filières les plus prestigieuses " (Baudelot \& Establet, op. cit., p 155). L'orientation aspirée vers l'apprentissage salarié résulte au final d'un processus de socialisation enseignant de "transformation" (Darmon, op. cit. $)^{16}$ qui modifie, en cours de scolarité, ses aspirations scolaires et contribue à produire ses dispositions prudentes. Chez Mirela, le sens conféré à cette orientation vient d'un soulagement de n'avoir plus à affronter seule et à l'avenir les matières scolaires théoriques.

16. Une telle socialisation fait subir une transformation aux individus, en général dans un seul domaine de pratique (ici au niveau des aspirations), selon un degré limité (légèrement revues à la baisse), alors qu’une socialisation de conversion implique une transformation plus étendue (dans plusieurs domaines) et beaucoup plus radicale. 


\section{Socialisation professionnelle partielle et revendication 3 d'une « identité pour soi d'apprenti salarié »}

Si l'orientation désirée vers l'apprentissage salarié tire notamment du discours enseignant d'éducation à l'orientation son caractère " d'évidence " ou "d'inéluctabilité ", ce projet se trouve au final renforcé, chez la majorité des aspirants apprentis de notre échantillon, lors de la réalisation des stages sur temps scolaire. L'entrée en stage figure ainsi comme une première étape provisoire, préparatoire ou de substitution - forme de socialisation préprofessionnelle - d'une éventuelle socialisation professionnelle plus large qui devrait débuter ensuite de manière formelle à l'entrée en formation et s'étendre jusqu'à l'exercice d'une profession, bien au-delà de l'entrée dans la vie professionnelle (Baszanger, 1981). Évoquer la notion de socialisation préprofessionnelle en se référant à Beaud \& Pialoux (2003), c'est considérer le côté "transitoire ", mais aussi « incomplet» d'une socialisation anticipée au travail et à l'occupation d'une position subalterne. S'y transmettent alors les premiers éléments d'une culture professionnelle où les jeunes acquièrent (ou renforcent) certaines "valeurs, attitudes, habiletés et savoir - en un mot la culture - qui sont ceux des groupes dont ils soubaitent devenir membres" (Merton, in Darmon, op cit., p. 76).

Pour les garçons, cette socialisation préprofessionnelle s'apparente à une première étape provisoire d'une socialisation à la condition ouvrière (sa culture, ses valeurs). Chez les apprentis, cette socialisation préprofessionnelle s'accentue et opère par une confrontation concrète et durable au travail en entreprise où se vivra une position objective qui n'est plus, comme chez les stagiaires, subjective, ni rêvée, et où s'exploreront les futures conditions de travail et d'emploi (Kergoat, 2006). Au final, cette socialisation préprofesionnelle peut être considérée comme "peu formalisée " (Dubois, 1999). En effet, elle se situe en amont (ou en substitut) de l'apprentissage salarié (qui s'effectue en école et en entreprise) et elle est, comme on va le voir, essentiellement définie dans la pratique.

La première sous-partie (3.1) aborde le rapport au travail salarié engagé par les élèves en stage et les dispositions qu'ils intériorisent ou renforcent lors du processus de découverte et d'acquisition des premiers éléments d'une culture professionnelle ; la seconde (3.2) présente les modalités spécifiques de cet apprentissage peu formalisé, où l'acquisition des savoirs métiers s'oppose à celle liée à la forme scolaire. 


\subsection{Un rapport positif au travail salarié}

Ce qui caractérise la majorité (sept sur neuf) des aspirants apprentis de notre échantillon, en particulier dans les secteurs secondaire (électriciens, mécaniciens, horlogers) et tertiaire du domaine des soins (assistantes en pharmacie/médicale) ${ }^{17}$, c'est le plaisir qu'ils éprouvent à entrer en stage, qui révèle "un rapport positif au travail " (Kergoat, op. cit., p. 550 $)^{18}$. Les activités réalisées offrent aux garçons un travail où les performances physiques - au contact de la matière - sont valorisées et où la reconnaissance est médiatisée par la capacité à se dépenser dans une tâche sans écouter sa fatigue. Á l'instar des ouvriers adultes, les garçons y acquièrent une "dignité par l'endurance physique devenue propriété morale " (Schwartz, 2002, p. 291), comme le suggère Dorian, qui a effectué un stage en électricité :

"J'étais en stage, on m'a dit "mais si tu viens électricien, il faut bien faire ton choix parce qu'il faut beaucoup se bouger, t'auras mal au dos, à cause d'être sur une échelle et puis, il faut tenir les câbles". Ça me fait pas peur. Ça me motive encore plus. "

La valorisation des activités tient à la fois au goût du défi de production (Kergoat, op. cit. $)^{19}$ qu'elles génèrent, mais aussi au besoin d'être en mouvement, de développer des dispositions à la débrouille :

"Le défi, j’aimerais si je fais ce travail, me lancer sur un truc et finir le plus vite possible, pour me tester moi-même. " (Dorian)

"Pour chaque voiture, on a un temps précis, on doit pas faire plus, le temps est chronométré, (...) je trouvais pas mal, l'esprit du challenge. " (Marc, stagiaire en mécanique automobile)

Les garçons parviennent à mobiliser des dispositions manuelles et au mouvement, intériorisées dans le milieu familial où leurs sont prêtées des qualités associées au sexe masculin, telles que le "besoin de se défouler" (Octobre, 2010). Cela suggère que la socialisation professionnelle est constamment pénétrée par des éléments qui ont leur origine ailleurs (Baszanger, op. cit.). Quant aux filles, au contact de la clientèle, elles ont

17. Á l'opposé, semble-t-il, des secteurs de la finance ou de la vente où deux aspirantes apprenties ont rapporté avoir vécu des expériences plus négatives (travail trop solitaire ou fatigue physique due au fait de rester debout toute la journée).

18. Un seul sur sept affiche un sentiment ambivalent où se mêlent plaisir à découvrir le métier de mécanicien et sentiment que les patrons ne donnent pas leur chance aux élèves en difficultés scolaires et exploitent les stagiaires, préférant engager ce genre de profils plutôt que des apprentis qu'ils n'ont ainsi pas à payer.

19. L'auteur fait ici brièvement référence à l'ouvrage de Burawoy, "Manufacturing Consent", pour parler des défis de production que se lancent et relèvent les ouvriers de l'industrie et qui témoignent d'un haut niveau d'engagement et de consentement dans une forme de travail qui reste subalterne. Si les patrons sont les principaux bénéficiaires des profits générés par cette productivité importante, cet engagement permet aux apprentis ouvriers de l'enquête de Kergoat de "participer, obtenir une reconnaissance et s'affirmer comme membre à part entière d'un groupe de pairs" (Kergoat, op. cit.). 
l'occasion d'y renforcer (ou d'intérioriser) des dispositions relationnelles, intériorisées vraisemblablement dans le milieu familial où, au sein des classes populaires, s'opère une valorisation précoce des attributs de la féminité (Octobre, op. cit.), ce que souligne Mirela, stagiaire en pharmacie :

"Quand jétais en stage, j'avais de très bons rapports avec les clients. Je suis toujours souriante, c'est important, parce que j'ai vu, elles [les clientes] disaient "elle est encore là l'autre blonde [stagiaire] ?" Ils ont dit "non, elle fait pas"... "heureusement, elle me servait, franchement, elle était pas souriante"."

\subsection{Un apprentissage par mimesis}

L'entrée en stage est vécue le plus souvent sur un mode "enchanté »" ${ }^{20}$ Le stage devient - à l'instar des ouvriers spécialisés plus âgés entrant en poste de travail (Lahire, 1993) - l'occasion, pour les aspirants apprentis, d'un apprentissage sur le tas en imitant ceux qui sont déjà là. S’opère alors un apprentissage de manière "diffuse par mimesis (voir-fairelfaire) et dans l'attention développée dans la pratique effective du travail» (ibid., p. 35), où les relations interpersonnelles jouent un rôle prépondérant.

Les explications fournies par les professionnels qualifiés sont des monstrations pratiques où le langage fonctionne comme marqueur de l'action. Dorian l'explicite :

" [Le patron] il m'a dit "tu prends un tournevis et tu dévisses un peu le truc en bas la cuisinière pour le câble" et puis c'est moi qui ai changé le câble et remis."

La réalisation du stage constitue également un moment propice pour les jeunes. Il leur permet de comparer les savoirs métiers (jugés " utiles » " intéressants ») appris dans l'activité (" où l'on " touche " " fait » et « voit le produit de son travail ») aux savoirs liés à la "forme scolaire » se définissant par un savoir décontextualisé, parlé et non pratiqué (Lahire, ibid.), ainsi que par un apprentissage séparé de la pratique (Cayouette, op. cit.), et qui sont fortement critiqués, comme l'explique Mirela :

"Je veux apprendre comment on fait le travail parce quà l'école, on fait pas trop de pratique, c'est surtout la théorie. C'est pour ça que je cherche beaucoup de stages, je trouve tout ce que je peux pas avoir à l'école. "

20. Si domine, dans les propos de six jeunes de notre échantillon, l'enchantement lié à la réalisation du travail, ceci tient sans doute à la fois au contexte (sur temps scolaire) dans lequel ils découvrent les métiers, mais aussi à la courte durée (chaque stage dure normalement une semaine) pendant laquelle ils sont exposés au travail physique : au plaisir au travail des apprentis facteurs et à l'excitation des jeunes travailleurs à l'usine, liés au fait d'être actifs, succèdent, après les premiers mois, un fort désenchantement des premiers (Kergoat, op. cit.) et une désillusion des seconds (Willis, op. cit.) lorsqu'ils réalisent la nature des tâches à effectuer (répétition, pénibilité). 
L'apprentissage par la pratique, celle-ci étant également présente dans de rares ateliers cuisine à l'école, devient l'occasion d'un agir qui s'oppose à la posture passive d'écoute liée à la « forme scolaire" :

Dorian : "Je faisais du saumon (... je trouvais génial, autre chose que toujours en classe, assis sur un bureau et puis écouter la prof pendant une heure."

Contrairement à ce qui se passe en classe, en stage, les jeunes ont l'occasion de voir, puis de mettre en œuvre par la pratique ce qui a été observé :

" [Le prof d'école] il peut nous expliquer, mais on peut pas voir....[à l'atelier] il peut nous expliquer comment enlever une bougie, il faut prendre le cricket et enlever, ben dire si on voit ça, je retiens plus vite[en tête]. " (Felippe, stage mécanique automobile)

C'est à l'occasion de ces stages que la majorité des élèves de notre échantillon, la plupart en "grandes difficultés scolaires ", confirment leur choix " idéal " de devenir apprenti. Se poursuit ici également le processus d'intériorisation du sens de leur place, puisqu'ils sont confrontés aux jugements des maîtres de stages, patrons et collègues de travail qui attribuent une nouvelle assignation identitaire renforcant celle attribuée par les discours enseignants et de certains parents :

"Les professionnels ont trouvé que jétais super bien, ils ont dit "tu pourrais faire une bonne assistante en pharmacie”." (Mirela)

"Le patron m'a dit "je sais pas ce que tu leur as fait [collègues], mais tout le monde a fait de bons rapports sur toi"." (Dorian)

Ainsi, les stages sont l'occasion d'une identification progressive au rôle, où s'élabore l'anticipation d'une trajectoire d'emploi par identification aux membres d'un groupe de référence (Dubar, op. cit.).

\section{Désirs et réalités : des aspirants apprentis contrariés}

L'expérience du stage renforce certes le choix désiré d'entrer en apprentissage salarié. Cependant, l'échec aux tests d'aptitude, le stigmate que semble constituer la filière B dans l'accès à la formation professionnelle (Hupka \& al. 2011), ainsi que les faibles résultats scolaires en fin d'année ${ }^{21}$, expliquent les réponses négatives des entreprises aux candidatures envoyées par les jeunes. Tout cela, couplé à de probables pratiques de discrimination à l'embauche touchant fortement les jeunes issus de la migration extra-européenne en

21. Seuls deux aspirants à l'apprentissage ont des « difficultés moyennes " contre sept qui sont en "grandes difficultés ». 
Suisse $^{22}$ (Fibbi, 2006) aboutit, pour la majorité des élèves de notre échantillon (sept sur neuf), à la non-conclusion du contrat d'apprentissage en fin de scolarité obligatoire.

La participation aux conseils de classe permet de saisir les orientations finalement réalisées par les aspirants à l'apprentissage salarié et de mesurer si les efforts fournis se sont matérialisés en projets réalisés. Ainsi, une minorité d'élèves $(\mathrm{N}=2)$ - d'origine suisse et portugaise - parvient à entrer directement en apprentissage. Toutefois, la majorité est " contrainte " de poursuivre, soit dans la voie scolaire à plein temps $(\mathrm{N}=5$ en préparatoire à l'ECG ou entrée en ECG lorsque le domaine visé n'existe pas sous forme d'apprentissage scolarisé, ou apprentissage scolarisé lorsqu'il existe), soit d'entrer au Centre de transition professionnelle (CTP) $(\mathrm{N}=2)$.

D’autres travaux quantitatifs ont montré, à Genève, chez les élèves des filières $\mathrm{B}$, « des projections à court terme souvent contredites par la réalité deux mois plus tard " (Rastoldo \& al., 2005, p. 25). Ceux qui aspirent à entrer au gymnase ou en apprentissage salarié sont particulièrement touchés. Les auteurs en déduisent une "importante dimension de frustration " (ibid., p. 26) à laquelle s'ajoute une dimension de «contrainte " qui fait qu' ils "apparaissent fortement tributaires de conditions extérieures qu'ils ne maîtrisent pas (marché de l'apprentissage, normes d'admission dans les écoles)" (ibid., p .26).

Ces résultats valident la thèse d'une complexification dans le temps des parcours des élèves des filières B. En effet, depuis les années 1990, et suite à la crise économique ayant engendré un déficit permanent de places d'apprentissages (Meyer, op. cit.), ces élèves entrent de moins en moins directement en apprentissage et de plus en plus souvent en structures d'insertion (DIP, 2009) ; et ce, dans un canton qui enregistre le plus faible taux d'entreprises formatrices et connaît la plus faible proportion de jeunes qui, dès la sortie de l'école obligatoire, entrent directement en formation professionnelle. Cette situation est liée non seulement aux exigences de cette formation, renforcées par l'évolution des technologies (Rastoldo \& al,. 2007), mais aussi au recrutement sélectif, par les patrons, de candidats plus âgés ${ }^{23}$. Cette situation semble d'ailleurs avoir (encore ?) peu évolué suite à l'incitation politique du Conseil d'État de créer des places d'apprentissage. Ainsi, l'entrée en apprentissage salarié est restée stable suite à la réforme scolaire (avec, de 2014 à 2016, un léger recul des entrées en formation générale au profit d'une entrée en formation professionnelle en école, cf. Rastoldo \& Mouad, 2017).

Le système scolaire engendre paradoxalement de la frustration, temporaire, pour certains élèves. En effet, les enseignants incitent directement ou indirectement les jeunes - en mobilisant des chiffres pour les motiver à s'inscrire au $\mathrm{CTP}^{24}$ - à s'orienter vers une formation

22. Cinq aspirants apprentis sur neuf ont une origine migratoire d'un pays extra-européen.

23. L'âge médian à l'entrée en apprentissage est passé, ces 25 dernières années, de 16 ans et demi à 18 ans (DIP 2015).

24. Conscients que nombre d'élèves n'ont que peu de chances, au vu de leurs notes, d'entrer directement en apprentissage, les enseignants tentent de revaloriser l'orientation vers le CTP, qu'ils préferent souvent à 
courte qui reste, en Suisse romande, moins valorisée que les formations académiques. Cependant, chez les "aspirants (apprentis) contrariés" (Orange, op. cit.), cette frustration varie selon les notes des élèves et le type d'apprentissage visé.

Ainsi, ceux qui ont des notes suffisantes acceptent, sans trop rechigner, de passer du statut " apprentissage salarié " à " apprentissage scolarisé " dans un domaine similaire, à l'instar de Manuel, qui s'oriente au final vers un apprentissage scolarisé d'horloger ("Je vais au CFPT, pas de problèmes "). Les élèves qui comptaient sur le salaire d'apprenti pour accéder à un mode de vie consommatoire ou pour échapper à la forme scolaire (comme Mirela), lorsqu'il n'existe pas d'apprentissage scolarisé dans le domaine visé, vivent l'orientation à l'ECG (souvent proposée aux filles par les enseignants en attendant de trouver une place d'apprentissage) comme une contrainte. Enfin, ceux qui ont des notes insuffisantes vivent la contrariété d'avoir à accepter la seule orientation possible, perçue comme très disqualifiante, au CTP, comme l'explique Daniel, qui fulmine :

"C'est une école de cons, ça je l'ai entendu, des copains qui reviennent, "putain, c'est de la merde!". Les gens qui vont là-bas foutent la merde. Une perte de temps, on doit reprendre une année, j'irai jamais là-dedans."

Ces propos révèlent le paradoxe qui fait qu'une partie des ressources pédagogiques dont sont privés les élèves des filières $B$ (programmes scolairement moins ambitieux effectivement dispensés, attentes professorales scolaires moindres, cf. Felouzis $\&$ al., 2010) "doivent être rattrapées par la suite» (Meyer, op. cit., p. 57).

\section{Conclusion}

Notre enquête montre ainsi que les choix scolaires ne peuvent être uniquement appréhendés à l'aune d'un "certain nombre de points du cursus", comme le pense Boudon (1984, p. 108). Les choix scolaires se construisent progressivement et dans la durée, en amont des orientations, lors des cours d'éducation à l'orientation à l'école, dès le début de la $11^{\mathrm{e}}$ année.

En effet, les enseignants contribuent, à l'instar de ce que révèlent les études réalisées en France, à " ajuster les espérances des élèves aux chances perçues" (Cayouette, op. cit.). D’une part, ils préparent non seulement les élèves des filières B (préprofessionnelles) souvent en grandes difficultés scolaires - à entrer directement ou indirectement en apprentissage salarié dans un contexte de revalorisation de cette formation duale, mais façonnent aussi, chez beaucoup, une "identité de travailleur salarié " par des exercices

l'option de la préparatoire à l'ECG/école de commerce parce que les élèves y sont "soutenus ", ne risquent pas de « rupture scolaire ». Pour motiver les élèves réticents à s'y inscrire, ils avancent le fait qu'à son terme, $70 \%$ d'entre eux en sortent avec un projet professionnel concret (un pourcentage surévalué semble-t-il, puisque $49 \%$ se trouvent en formation à la fin du CTP, cf. Hrizi \& al., op. cit.). 
quotidiens en classe ou la recherche de stages. D'autre part, en anticipant un échec ultérieur, ils omettent d'évoquer la possibilité de viser une entrée au gymnase, au profit d'une entrée à l'ECG pour préparer des réorientations ultérieures vers l'apprentissage salarié, dont les exigences en termes de niveau de qualification se sont accrues.

Dès lors, les enseignants participent à l'anticipation de l'avenir objectif, mais aussi à la production ou au renforcement d'un "sentiment d'évidence" (Orange, op. cit.) également produit dans l'environnement familial et, in fine, à l'auto-exclusion des études longues pour les fractions les moins diplômées des jeunes des classes populaires.

Les enseignants qui s'arrogent davantage la responsabilité de l'orientation à Genève qu'en France (Boudon, 1990), exercent ainsi, selon les cas, une influence socialisatrice de "renforcement " ou de "transformation" (Darmon, op. cit.) des aspirations juvéniles et participent d'une socialisation juvénile anticipée au statut d'apprenti. Dès lors, et à l'instar de Bourdieu, il convient de prendre au sérieux les rappels à l'ordre qu'impose le système scolaire ou qui surgissent "des interactions de l'existence ordinaire " et qui conduisent à "inscrire progressivement l'ordre social dans les cerveaux" (Bourdieu, op. cit., p. 549).

Le sens des limites se forge ensuite (et se renforce) au contact du monde professionnel, lors des stages sur temps scolaire durant le $2^{\mathrm{e}}$ trimestre. Les maîtres de stages initient ces élèves au monde du "travail salarié ", davantage qu'en France où la formation professionnelle se fait plus souvent sous statut scolaire. Cette initiation a lieu lors d'un processus de socialisation préprofessionnelle au cours duquel les élèves expérimentent souvent un rapport positif au travail et renforcent (ou intériorisent), par un apprentissage sur le tas et dans la pratique (Lahire, op cit.), diverses dispositions (à la débrouille, manuelles, à la résistance à la douleur, relationnelles) qui, dans certaines conditions, peuvent faire "ressources" dans un parcours de difficultés sociales, mais qui contribuent dans le même temps à enfermer (Thin \& Faure, 2019). En produisant des verdicts positifs permettant à ces jeunes en difficultés scolaires de se projeter de manière concrète dans les métiers subalternes et de confirmer le choix idéal d'entrer en apprentissage, les maîtres de stages, patrons et collègues de travail - acteurs souvent peu considérés des études de cas qui se concentrent sur le travail des institutions scolaires et familiales (Palheta, op. cit. ; Cayouette, op. cit.) - jouent le rôle de socialisation de renforcement par rapport aux socialisations, scolaire et familiale. Le dévouement au travail manuel, manifesté en particulier par les garçons de notre échantillon, indique en outre, et à la suite des travaux de Kergoat (op cit.), que pour une certaine fraction des jeunesses des classes populaires, l'entrée (temporaire) dans le monde ouvrier, par le biais de stages scolaires, s'effectue sur d'autres modes que celui présenté par Beaud \& Pialoux (op. cit.) de la désaffection à l'égard du travail ouvrier.

Les choix idéaux se confrontent également, à la fin de l'année scolaire, aux orientations finalement réalisées. Dès lors, cette confrontation permet de saisir les difficultés rencontrées dans leur réalisation et de mettre en évidence, à l'instar des travaux d'Orange 
(op. cit.), un important groupe "d'aspirants contrariés", que des enquêtes cherchant à saisir les choix de manière rétrospective tendent à ne pas voir. La frustration qui en découle, temporaire et variable pour certains, est paradoxalement générée par un système scolaire qui cherche à promouvoir, chez les bons élèves des filières $\mathrm{B}$, une entrée directe vers l'apprentissage salarié pour éviter les réorientations ultérieures (du gymnase vers les autres formations : Felouzis $\&$ al., 2010). Il fait ainsi miroiter, aux élèves scolairement plus fragiles, qu'une telle orientation directe est possible et souhaitable, alors qu' elle reste - et les enseignants en sont conscients - très marginale dans les faits, dans un contexte de pénurie de places d'apprentissage pour les élèves en difficulté et d'une économie genevoise fortement tournée vers un secteur tertiaire exigeant en termes de qualifications. L'école participe ainsi à produire, chez ces élèves, un «sens de la place» et de "l'orientation" fortement contrarié (du moins à court terme).

Pour tenter de minorer cette frustration collective, les autorités politiques genevoises se sont engagées à mettre en place, pour la période 2015-2018, des mesures visant à assurer des transitions plus fluides entre l'école obligatoire et le monde des entreprises : augmentation du nombre de places d'apprentissage (par exemple employés de commerce) au sein de l'État ${ }^{25}$, dans certains départements qui emploient du personnel administratif et technique, abaissement de l'âge d'entrée en formation duale, prospection des entreprises pour les inciter à développer une offre d'apprentissage de courte durée (AFP), peu étoffée à Genève, ou encore création d'équipes de conseillers en insertion dans certains établissements du secondaire inférieur, afin de proposer aux entreprises des dossiers d'élèves au projet professionnel défini (DIP, 2015).

Si de telles mesures permettent d'aider les jeunes dans la transition de l'école obligatoire vers la formation secondaire supérieure, elles pourraient demeurer insuffisantes au regard des difficultés spécifiques que rencontrent les élèves d'origine étrangère à intégrer le marché de l'apprentissage. Pour progresser sur le chemin de l'égalité, il paraît impératif de développer également des mesures du côté des entreprises. Elles pourraient consister à inciter les formateurs potentiels à s'intéresser aux candidatures des jeunes d'origine étrangère et à accorder plus de poids aux résultats qu'au type de filière suivie, de même qu’à surmonter certains préjugés et craintes liés aux groupes "présumés problématiques» (voir aussi Imdorf, 2007).

25. Il s'agit ici de l'administration publique fiscale, mais aussi des établissements publics autonomes tels que les Hautes écoles, l'Hôpital, l'Office cantonal des assurances... 
Ainsi, cette étude contribue aux réflexions permettant de "favoriser l'intégration professionnelle et l'inclusion sociale ${ }^{26}$ des élèves en difficultés scolaires. En effet, à la fin de la scolarité obligatoire et dans un contexte mouvant, elle identifie leurs besoins et les obstacles contextuels spécifiques que certains rencontrent.

\section{Bibliographie}

Baudelot C. \& Establet R. (1992), Allez les filles!, Paris, Seuil.

Baszanger I. (1981) «Socialisation professionnelle et contrôle social. Le cas des étudiants en médecine futurs généralistes ", Revue française de sociologie, 22, pp. 223-245.

Beaud S. \& Pialoux M. (1999), Retour sur la condition ouvrière, Paris, Seuil.

Beaud S. \& Pialoux M. (2003), Violences urbaines, violence sociale. Genèse des nouvelles classes dangereuses, Paris, Hachette.

Blanchard M. \& Cayouette-Rembliere J. (2011), " Penser les choix scolaires ", Revue française de pédagogie, $\mathrm{n}^{\circ} 175$, pp. 5-14.

Bolzman C. \& Perregaux C. (2008), "Quelles chances d'accès aux formations professionnelles pour les jeunes étrangers en Suisse ? ", in Hanhart S. \& al. (Eds.), De la comparaison en Education?, Paris, l'Harmattan, pp. 201-222.

Bolzman C., Fibbi R. \& Vial M. (2003), Secondas, secondos : le processus d'intégration des jeunes adultes issus de la migration espagnole et italienne en Suisse, Zurich, Seismo.

Boudon R. (1984), L'inégalité des chances, Paris, Hachette Littérature.

Boudon R. (1990), "Les causes de l'inégalité des chances scolaires ", Conférence pour la Fondation Saint-Simon, 12 février.

Bourdieu P. (1980), Le sens pratique, Paris, Minuit.

Bourdieu P. (1979), La Distinction, Paris, Minuit.

Bourdieu P. \& Passeron J.-C. (1985) [1964], Les héritiers, Paris, Minuit.

26. Selon Di Maggio, Santilli, Ginevra, Martella \& Nota (2019), qui défendent une « approche inclusive de l'orientation", favoriser l'inclusion signifie transformer les communautés en lieux conviviaux pour toutes et tous. La lutte pour l'inclusion exige que tous les individus et que toutes les institutions jouent un rôle actif pour faciliter la participation de chacun.e dans la vie sociale. Le contexte joue un rôle décisif pour favoriser cette inclusion et les difficultés rencontrées dans la vie sociale par les individus peuvent être réduites en "modifiant les exigences contextuelles et en apportant un soutien pour faciliter la participation" (ibid., p. 209). Les auteurs soulignent, par exemple, le besoin d'impliquer les employeurs pour soutenir l'insertion professionnelle des personnes en situation de handicap. 
Brinbaum Y., Kieffer A. (2005), « D’une génération à l'autre, les aspirations éducatives des familles immigrées : ambition et persévérance ", Éducation \& Formations, $\mathrm{n}^{\circ} 72$, pp. 53-75.

Cayouette-Rembliere J. (2016), L'école qui classe, Paris, PUF.

Chauvel S. (2011), "Auto-sélections et orientation en fin de $3^{\mathrm{e}}$ : réflexions issues d'une enquête de terrain ", Revue française de pédagogie, n 175, pp. 85-88.

Darmon M. (2006), La socialisation, Paris, Armand Colin.

Di Maggio I., Santilli S., Ginevra M.-C., Martella G. \& Nota L. (2019), «Inclusion, Life design, coaching de carrière et handicap ", in Masdonati J., Massoudi K. \& Rossier J. (dir.), Repères pour l'orientation, Lausanne, Antipodes, pp. 207-231.

Delay C. (2018), “"En sortir” ou “s'en sortir” par l'école. Choix d'orientations juvéniles, classes populaires et enseignement professionnel en Suisse romande ", Sociétés contemporaines, $\mathrm{n}^{\circ} 110$, pp. 117-147.

Dip (2015), Soutenir et valoriser l'apprentissage dans le canton de Genève. Plan d'action du conseil d'État 2015-2018, Genève.

Dip (2011 et 2009), L'enseignement à Genève. Indicateurs du système genevois de formation, Genève, SRED.

Dubar C. (2010), La socialisation. Construction des identités sociales et professionnelles, Paris, Armand Colin.

Dubois V. (1999), La vie au guichet, Paris, Economica.

Duru-Bellat M. (2002), Les inégalités sociales à l'école, Paris, PUF.

Duru-Bellat M. (2008), « La (re)production des rapports sociaux de sexe : quelle place pour l'institution scolaire?", Travail, genre et société, 19, pp. 131-149.

Fibbi R. (2006), "Discrimination dans l'accès à l'emploi des jeunes d'origine immigrée en Suisse ", Formation Emploi, n 94, pp. 45-58.

Felouzis G. \& Goastallec G. (2015), Les inégalités scolaires en Suisse, Berne, Peter Lang.

Felouzis G., Charmillot S., Fouquet-Chauprade B. (2010), L'enseignement secondaire au risque des enquêtes PISA Suisse. Parcours scolaires, performances et orientation à Genève, Rapport de recherche, Université de Genève.

Gomensoro A. \& Bolzman C. (2015), "The effect of socioeconomic status of ethnic groups on educational inequalities in Switzerland: which hidden mechanisms?", Italian journal of sociology of education, $\mathrm{n}^{\circ} 7(2)$, pp. 70-98.

Guilley E., Arruda C., Gauthier J., Gianettoni L, Gross D., Joye D. \& al. (2014), Maçonne ou avocate : rupture ou reproduction sociale? Une enquête sur les aspira- 
tions professionnelles des jeunes en Suisse, Genève \& Lausanne, SRED \& Université Lausanne.

Hupka-Brunner S., Sacchi S. \& Stalder B. (2011), "Social origin and access to upper secondary education in Switzerland: a comparison of company-based apprenticeship and exclusively School-based programmes", in Bergman M. \& al., Transitions juvéniles en Suisse, Zurich, Seismo, pp. 157-182.

Hrizi Y., Mouad R., Petrucci F. \& Rastoldo F. (2014), « Les parcours de formation des jeunes en difficultés scolaires à la fin du cycle d'orientation ", Note d'information $d u$ SRED n ${ }^{\circ}$ 65, pp. 1-8.

Imdorf C. (2007), "La sélection des apprentis dans les PME ", Institut de pédagogie curative de l'Université de Fribourg, pp. 1-8.

Kergoat P. (2006), «De l'indocilité au travail d'une fraction des jeunesses populaires. Les apprentis et la culture ouvrière ", Sociologie du travail, 48, pp. 545-560.

Korber M. \& Oesch D. (2016) «Quelles perspectives d'emploi et de salaire après un apprentissage?", Social change in Switzerland, 6, pp. 1-16.

Lahire B. (1993), La raison des plus faibles, Paris, PUL.

Lahire B. (2002), Portraits sociologiques, Paris, Nathan.

Lemaire S. (1996), "Qui entre en lycée professionnel, qui entre en apprentissage ? Profils d'élèves qui intègrent une filière professionnelle à l'issue du collège ", Éducation \& Formations, $n^{\circ} 48$, pp. 71-80.

Mauger G. (1991), "Enquêter en milieu populaire », Genèses, n 6, pp. 125-143.

Meyer T. (2011), "On ne prête qu'aux riches : l'inégalité des chances devant le système de formation en Suisse ", in Bergman M. \& al. (éd.) Transitions juvéniles en Suisse, Zurich, Seismo, pp. 40-65.

Meyer T. (2009), "Can vocationalisation of education go too far? The case of Switzerland”, European journal of vocational training, 46(1), pp. 28-40.

Moreau G. (2003), Le monde apprenti, Paris, La Dispute.

Octobre S. (2010), "La socialisation culturelle sexuée des enfants au sein de la famille ", Cahiers du genre, n 49(2), pp. 55-76.

Office Fédéral de la Statistique (2018), Transitions après un titre du degré secondaire II et intégration sur le marché du travail, Neuchâtel.

Orange S. (2013), L'autre enseignement supérieur. Les BTS et la gestion des aspirations scolaires, Paris, PUF.

Palheta U (2012), La domination scolaire, Paris, PUF. 
Pasquali P. (2010), "Les déplacés de 'l'ouverture sociale' ", Actes de la recherche en sciences sociales, $\mathrm{n}^{\circ} 183(3)$, pp. 86-105.

Rastoldo F. \& Mouad R. (2017), L'enseignement à Genève : repères et indicateurs statistiques, Genève, SRED.

Rastoldo F., Evrard A. \& Amos J. (2007), Les jeunes en formation professionnelle, Genève, SRED.

Rastoldo F., Kaiser C., Alliata R. (2005), La nouvelle organisation du cycle d'orientation, Genève, SRED.

Schwartz O. (2002), Le monde privé des ouvriers, Paris, PUF.

Thin D. \& Faure S. (2019), "Du discours sur la vulnérabilité aux ressources des classes populaires ", in Faure S. \& Thin D. (Dir.), S'en sortir malgré tout. Parcours en classes populaires, Paris, La Dispute, pp. 9-25.

Willis P. (2011)[1977], L'école des ouvriers, Paris, Agone.

Wolter S. (2018, 2014), Swiss Education Report, Aarau, Swiss coordination centre for Research in Education.

Zunigo X. (2008), "L'apprentissage des possibles professionnels ", Sociétés contemporaines, vol. $2, \mathrm{n}^{\circ} 70$, pp. $115-131$. 